\title{
Risk of Oral Contraceptives and Recency of Market Introduction
}

\section{To the Editors:}

In the recent exchange of views on the impact of the epidemiologic studies on deep vein thrombosis and third generation contraceptives, ${ }_{1}^{1,2}$ the earlier publication by Lewis et al. from the Transnational Study ${ }^{3}$ plays an important role. It indicated that the risks increase according to the recency of market introduction of the oral contraceptive. ${ }^{3}$ However, the important Figure 1, which graphically shows increasing odds ratios by year of introduction, was based only on the subset of women aged $25-44 .^{3}$ The data of the women aged 16-24 were left out, which amounts to one-third of the cases and close to half of the controls who used oral contraceptives. The same publication contains data and odds ratios for all ages (16-44), wherein no trend is apparent. The authors stated that
Table. Relative risk of venous thromboembolism in women aged 16-24 years; data recalculated from published tables of the Transnational Study ${ }^{3}$

\begin{tabular}{lrcc}
\hline & Cases & Controls & OR \\
\hline Levonorgestrel & 27 & 104 & 1.0 \\
Gestodene & 24 & 66 & 1.4 \\
Desogestrel $(30 \mu \mathrm{g})$ & 32 & 48 & 2.6 \\
Desogestrel $(20 \mu \mathrm{g})$ & 2 & 20 & 0.4 \\
Norgestimate & 8 & 18 & 1.7 \\
Other OC (oestradiol $<50 \mu \mathrm{gg})$ & 10 & 30 & 1.3 \\
OC with oestradiol $>50 \mu g$ & 6 & 12 & 1.9 \\
Progesterone-only pills (POP) & 4 & 8 & 1.9 \\
\hline
\end{tabular}

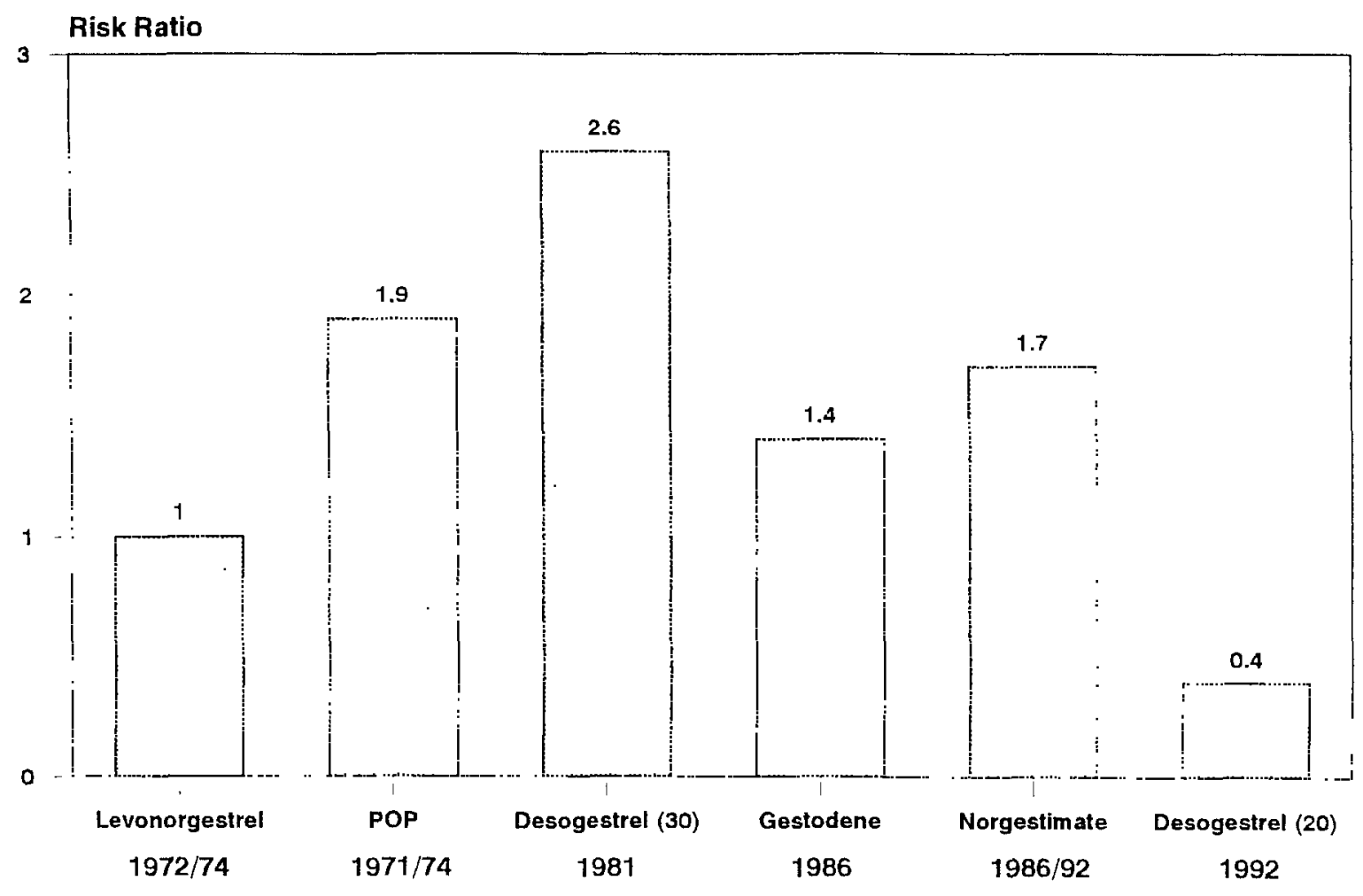

Type of oral contraceptive and year of market introduction

Figure. Risk ratios of combined oral contraceptives compared with levonorgestrel for women aged 16-24 by year of market introduction; recalculated from published data of Transnational Study. ${ }^{3}$ 
they had restricted their analysis to the 25-44 age bracket because among the younger women, too many would have been taking the newer pillsdespite their demonstration, further in the paper, that more than half of contraceptive-using women in the age group 16-24 used older types of pills.

Fortunately, it was possible to reconstruct the data for the ages 16-24 by subtracting the table for the ages 25-44 from the overall table (Tables 2 and 3), and recalculate the odds ratios for the ages 16-24. The data for the youngest women are the most relevant, since we expect most of the new users among them. When doing the same analyses as the authors, we find the following Table and Figure. The trend of the odds ratios among women aged 16-24 indicates an overall higher risk of third generation products, but almost an inverse relation with year of introduction. This denies the conclusion of the authors, and at the same time explains why the trend is not present in the complete data from the Transnational Study (Table 2). Moreover, inclusion of older types of pills in the figure-introduced before levonorgestrel-containing pills, and for which the recency argument does not hold-would lead to a V-shaped curve, indicating that the higher risks of older preparations have indeed returned.

While we greatly commend the authors of the Transnational Study for their open style of publication, which permits these recalculations, there is the distinct possibility that they have fallen into the trap of publishing the most pleasing subgroup analysis.

\section{References}

1. Farley TMM, Meirik O, Poulter NR, Chang CL, Marmot MG. Oral contraceptives and thrombotic diseases: impact of new epidemiologic studies (Jetter). Contraception $1996 ; 54: 193-5$

2. Lidegaard $\varnothing$, Milsom I. Response to the editor (letter). Contraception 1996;54:195-8.

3. Lewis MA, Heinemann LAJ, MacRae KD, Bruppacher R, Spitzer WO. The increased risk of venous thromboembolism and the use of third generation progestagens: role of bias in observational research. Contraception 1996;54: $5-13$

Jan P. Vandenbroucke Department of Clinical Epidemiology

Kitty W.M. Bloemenkamp Frans M. Helmerhorst

Department of Obstetrics Gynecology and Reproductive Medicine

Frits R. Rosendaal Thrombosis and Haemostasis Research Centre Leiden University Hospital Bldg-1

PO Box 9600 2300 RC Leiden The Netherlands

PII S0010-7824(97)00001-2

\section{RESPONSE TO THE EDITOR}

\section{The Role of Bias in Observational Studies on Oral Contraceptives}

We would like to thank Drs. Weiss ${ }^{1}$ and Vandenbroucke et $a l^{2}$ for their commentaries. These focus mainly on a criticism related to our using only selected portions of the data, namely data on women aged $25-44$, in our analysis. ${ }^{3}$ We will explain the rationale more clearly than was done in our article. This group was selected for two reasons. First, we wanted to have a group which is likely to have been exposed to all oral contraceptives on the market, including the older preparations. Second, the phenomenon of attrition of susceptibles (or, conversely, the existence of a stable group of "healthy users") could only be demonstrated in a group in which high-risk individuals have already been removed (i.e. a stable group of levonorgestrel users), or are in the process of being removed (i.e. groups taking newer proge- stagens|, which again leaves us with the older age group. Because our reference group was chosen to be levonorgestrel, we clearly need a population with exposures of sufficient duration to have formed a stable healthy user group. At the time of the analysis, we felt that this status can only occur in women past the age of 25, whose potential exposure experience ranges from about 10 years to 30 years.

This rationale has been confirmed empirically to be appropriate. A comparison of duration of use of two progestagens (levonorgestrel and gestodene) in controls stratified by the two age groups (Table) shows that duration of current use is almost the same in both age groups for gestodene, but about $30 \%$ of women aged 25-44 years were at the time of interview using levonorgestrel for over 6 years compared with only $8 \%$ of $16-24$ year old women. This means that there is an appreciably larger proportion of 